\title{
THE SET OF REGULAR VALUES (IN THE SENSE OF CLARKE) OF A LIPSCHITZ MAP. A SUFFICIENT CONDITION FOR THE RECTIFIABILITY OF CLASS $C^{3}$.
}

\author{
SILVANO DELLADIO
}

\begin{abstract}
Let $n, N$ be positive integers such that $n<N$. We prove a result about the rectifiability of class $C^{3}$ of the set of regular values (in the sense of Clarke) of a Lipschitz map $\varphi: \mathbb{R}^{n} \rightarrow \mathbb{R}^{N}$.
\end{abstract}

\section{IntRodUCtion AND STATEMENT OF MAIN RESUlt}

In this paper we prove a result about the rectifiability of class $C^{3}$ of the set of regular values (in the sense of Clarke) of a Lipschitz map

$$
\varphi: \mathbb{R}^{n} \rightarrow \mathbb{R}^{N} \quad(n<N) .
$$

Before we state it, let us recall some basic definitions.

A Borel subset $S$ of $\mathbb{R}^{N}$ is said to be an $\left(\mathcal{H}^{n}, n\right)$ rectifiable set of class $C^{3}$ (or simply: a rectifiable set of class $C^{3}$ ), if there exist countably many $n$-dimensional submanifolds $M_{j}$ of $\mathbb{R}^{N}$ of class $C^{3}$ such that

$$
\mathcal{H}^{n}\left(S \backslash \bigcup_{j} M_{j}\right)=0 .
$$

Analogously one can define the $\left(\mathcal{H}^{n}, n\right)$ rectifiable sets of class $C^{k}$, for each positive integer $k$. In particular, for $k=1$ this notion is equivalent to that of $n$-rectifiable set, e.g. by [S, Lemma 11.1].

For $\gamma \in I(n, N)$ and $s \in \mathbb{R}^{n}$, let $\partial \varphi^{\gamma}(s)$ denote the Clarke subdifferential of the map

$$
\varphi^{\gamma}:=\left(\varphi^{\gamma_{1}}, \ldots, \varphi^{\gamma_{n}}\right): \mathbb{R}^{n} \rightarrow \mathbb{R}^{n}
$$

namely

$$
\partial \varphi^{\gamma}(s):=\operatorname{co}\left\{\lim _{i \rightarrow \infty} D \varphi^{\gamma}\left(s_{i}\right) \mid D \varphi^{\gamma}\left(s_{i}\right) \text { exists, } s_{i} \rightarrow s\right\}
$$

compare [CLSW, p.133]. The set $\partial \varphi^{\gamma}(s)$ is said to be "nonsingular" if every matrix in $\partial \varphi^{\gamma}(s)$ is of rank $n$. Observe that $D \varphi^{\gamma}(s) \in \partial \varphi^{\gamma}(s)$ whenever $\varphi^{\gamma}$

2010 Mathematics Subject Classification. Primary 26A16, 49Q15, 53A04, 54C20; Secondary 28A75, 28A78, 49Q20.

Key words and phrases. Rectifiable sets, Geometric measure theory, Whitney extension theorem. 
is differentiable at $s$. In particular, $D \varphi^{\gamma}(s)$ is nonsingular provided $\partial \varphi^{\gamma}(s)$ is nonsingular. Define

$$
\mathcal{R}:=\left\{s \in \mathbb{R}^{n} \mid \partial \varphi^{\gamma}(s) \text { is nonsingular for some } \gamma\right\} .
$$

We can now state our theorem.

Theorem 1.1. Consider a Lipschitz map

$$
\varphi: \mathbb{R}^{n} \rightarrow \mathbb{R}^{N} \quad(n<N) .
$$

Moreover let

$$
c_{1, i}, c_{2, i}: \mathbb{R}^{n} \rightarrow \mathbb{R} \backslash\{0\} \quad(i=1, \ldots, n)
$$

be a family of locally bounded functions, let

$$
G_{1, i}, G_{2, i}: \mathbb{R}^{n} \rightarrow \mathbb{R}^{N} \quad(i=1, \ldots, n), \quad H_{i j}: \mathbb{R}^{n} \rightarrow \mathbb{R}^{N} \quad(i, j=1, \ldots, n)
$$

be a family of Lipschitz maps and denote by $A$ the set of points $t \in \mathbb{R}^{n}$ satisfying the following conditions:

(i) The map $\varphi$ and all the maps $G_{1, i}$ are differentiable at $t$;

(ii) The equality

$$
D_{i} \varphi(t)=c_{1, i}(t) G_{1, i}(t)=c_{2, i}(t) G_{2, i}(t)
$$

holds for all $i=1, \ldots, n$;

(iii) Moreover one has

$$
D_{j} G_{1, i}(t)=c_{2, j}(t) H_{i j}(t)
$$

for all $i, j=1, \ldots, n$.

Also assume that

(iv) For almost every $a \in A$ there exists a non-trivial ball $B$ centered at $a$ and such that

$$
\mathcal{L}^{n}(B \backslash A)=0 .
$$

Then $\varphi(A \cap \mathcal{R})$ is an $\left(\mathcal{H}^{n}, n\right)$ rectifiable set of class $C^{3}$.

Remark 1.2. As an immediate corollary of Theorem 1.1, we get this result. Let

$$
\varphi: \mathbb{R}^{n} \rightarrow \mathbb{R}^{N}, \quad G_{1, i}, G_{2, i}, H_{i j}: \mathbb{R}^{n} \rightarrow \mathbb{R}^{N}(i, j=1, \ldots, n)
$$

be a family of Lipschitz maps and let

$$
c_{1, i}, c_{2, i}: \mathbb{R}^{n} \rightarrow \mathbb{R} \backslash\{0\} \quad(i=1, \ldots, n),
$$

be a family of bounded functions such that the equalities (1.1) and (1.2) hold almost everywhere in $\mathbb{R}^{n}$. Then the image $\varphi(\mathcal{R})$ is an $\left(\mathcal{H}^{n}, n\right)$ rectifiable set of class $C^{3}$. 
Remark 1.3. Let each component $\varphi_{i}$ of $\varphi: \mathbb{R}^{n} \rightarrow \mathbb{R}^{N}$ belong to $C^{3}\left(\mathbb{R}^{n}\right)$ and have uniformly bounded gradient $\nabla \varphi_{i}$. Moreover let the differential $D \varphi$ have rank $n$ at each point of $\mathbb{R}^{n}$. Then the assumptions of Theorem 1.1 are trivially satisfied by setting

$$
c_{1, i}:=1, \quad c_{2, i}:=1, \quad G_{1, i}:=D_{i} \varphi, \quad G_{2, i}:=D_{i} \varphi \quad(i=1, \ldots, n)
$$

and

$$
H_{i j}:=D_{i j}^{2} \varphi \quad(i, j=1, \ldots, n)
$$

with $A=\mathbb{R}^{n}$.

Rectifiable sets of class $C^{k}$ have been been introduced in [AS] and provide a natural setting for the description of singularities of convex functions and convex surfaces, $[\mathrm{A}, \mathrm{AO}]$. More generally, it can be used to study the singularities of surfaces with generalized curvatures, [AO]. Rectifiability of class $C^{2}$ is strictly related to the context of Legendrian rectifiable subsets of $\mathbb{R}^{N} \times \mathbf{S}^{N-1}$, [Fu1, Fu2, D2, D3]. The level sets of a $W_{\text {loc }}^{k, p}$ mapping between manifolds are rectifiable sets of class $C^{k}$, [BHS]. Applications of rectifiable sets of class $C^{H}$ (with $H \geq 2$ ) to geometric variational problems can be found in [D4].

Finally, we would like to explain the reasons of our interest in conditions (1.1) and (1.2). In the particular case when $n=1$, such conditions arised naturally in the context of one-dimensional generalized Gauss graphs (see [AST, D1], for the basic definitions and results) and of two-storey towers of one-dimensional generalized Gauss graphs (see [D4]). After that it was natural to explore the question of how those assumptions could be generalized in order to get results about higher order of rectifiability, including the case when $n \geq 2$. Then a general theorem for curves was provided in [D3], while in [D5] we started studying the case of general dimension by proving a result about the rectifiability of class $C^{2}$. Further results in this direction can be found in [AS] and [Fu1, Fu2]. Roughly speaking, the very basic idea and the proof-strategy in the present paper are the same as in [D5] namely: to use the celebrated Whitney extension Theorem to show that the image of $\varphi$ is captured, up to $\mathcal{H}^{n}$ measure 0 , by countably many high regular images of $\mathbb{R}^{n}$. More precisely, the main objective of this work is to get the set of third order Whitney estimates which allows one to perform the (countably many) extensions of class $C^{3}$ necessary to show that the image of $\varphi$ is $C^{3}$-rectifiable. Such a result is the product of our efforts to prove a general theorem about rectifiability of class $C^{H}$ in any dimension, which will be the subject of our future further investigations. 


\section{REDUCTION TO GRAPHS}

Remark 2.1. Under the hypotheses of Theorem 1.1, let $A^{\prime}$ denote the set of $a \in A$ such that there exists a non-trivial ball $B$ centered at $a$ satisfying

$$
\mathcal{L}^{n}(B \backslash A)=0
$$

One has

$$
\mathcal{L}^{n}\left(A \backslash A^{\prime}\right)=0
$$

by assumption (iv). Hence, it will be enough to prove that $\varphi\left(A^{\prime} \cap \mathcal{R}\right)$ is an $\left(\mathcal{H}^{n}, n\right)$ rectifiable set of class $C^{3}$.

Remark 2.2. By the main theorem in [D5], we already know that $\varphi(A \cap \mathcal{R})$ (hence also $\left.\varphi\left(A^{\prime} \cap \mathcal{R}\right)\right)$ is an $\left(\mathcal{H}^{n}, n\right)$ rectifiable set of class $C^{2}$.

Remark 2.3. Let $E$ be any subset of $\mathcal{R}$ and define

$$
E^{\gamma}:=\left\{s \in E \mid \partial \varphi^{\gamma}(s) \text { is nonsingular }\right\}, \quad \gamma \in I(n, N) .
$$

Then one obviously has

$$
\bigcup_{\gamma \in I(n, N)} E^{\gamma}=E .
$$

Remark 2.4. If $s \in \mathcal{R}^{\gamma}$, by the Lipschitz inverse function Theorem (e.g. [CLSW, Theorem 3.12]), there exist a neighborhood $U$ (in $\mathbb{R}^{n}$ ) of $s$ and a neighborhood $V$ (in $\mathbb{R}^{n}$ ) of $\varphi^{\gamma}(s)$ such that

- $V=\varphi^{\gamma}(U)$ and $\varphi^{\gamma} \mid U: U \rightarrow V$ is invertible;

- $\left(\varphi^{\gamma} \mid U\right)^{-1}$ is Lipschitz.

Let $\bar{\gamma}$ denote the multi-index in $I(N-n, N)$ which complements $\gamma$ in $\{1,2, \ldots, N\}$ in the natural increasing order and set (for $x \in \mathbb{R}^{N}$ )

$$
x^{\gamma}:=\left(x^{\gamma_{1}}, \ldots, x^{\gamma_{n}}\right), \quad x^{\bar{\gamma}}:=\left(x^{\bar{\gamma}_{1}}, \ldots, x^{\bar{\gamma}_{N-n}}\right) .
$$

Then the map

$$
f:=\varphi^{\bar{\gamma}} \circ\left(\varphi^{\gamma} \mid U\right)^{-1}: V \rightarrow \mathbb{R}^{N-n}
$$

is Lipschitz and its graph

$$
\mathcal{G}_{f}^{\gamma}:=\left\{x \in \mathbb{R}^{N} \mid x^{\gamma} \in V \text { and } x^{\bar{\gamma}}=f\left(x^{\gamma}\right)\right\}
$$

coincides with $\varphi(U)$.

By virtue of previous remarks, it will be enough to prove the following claim. 
Theorem 2.5. Under the assumptions of Theorem 1.1, let $\gamma \in I(n, N)$ and consider a map

$$
g: \mathbb{R}^{n} \rightarrow \mathbb{R}^{N-n}
$$

of class $C^{2}$. Then $\varphi\left(\left(A^{\prime} \cap \mathcal{R}\right)^{\gamma}\right) \cap \mathcal{G}_{g}^{\gamma}$ is an $\left(\mathcal{H}^{n}, n\right)$ rectifiable set of class $C^{3}$.

Remark 2.6. The remainder of our paper is devoted to proving Theorem 2.5. With no loss of generality, we can restrict our attention to the particular case when $\gamma=\{1, \ldots, n\}$.

\section{Preliminaries}

3.1. Further reduction of the claim. From now on, for simplicity,

$$
\mathcal{G}_{g}^{\{1, \ldots, n\}}, \quad\left(A^{\prime} \cap \mathcal{R}\right)^{\{1, \ldots, n\}}, \quad \varphi^{\{1, \ldots, n\}}
$$

will be denoted by $\mathcal{G}_{g}, F$ and $\lambda$, respectively.

Define

$$
L:=\varphi^{-1}\left(\mathcal{G}_{g}\right) \cap F .
$$

Without loss of generality, we can assume that $\mathcal{L}^{n}(L)<\infty$. Then, by a well-known regularity property of $\mathcal{L}^{n}$, for any given real number $\varepsilon>0$ there exists a closed subset $L_{\varepsilon}$ of $\mathbb{R}^{n}$ with

$$
L_{\varepsilon} \subset L, \quad \mathcal{L}^{n}\left(L \backslash L_{\varepsilon}\right) \leq \varepsilon,
$$

compare e.g. [M, Theorem 1.10]. Moreover, since $L_{\varepsilon}$ is closed, one has

$$
L_{\varepsilon}^{*} \subset L_{\varepsilon}
$$

where $L_{\varepsilon}^{*}$ is the set of density points of $L_{\varepsilon}$. Recall that

$$
\mathcal{L}^{n}\left(L_{\varepsilon} \backslash L_{\varepsilon}^{*}\right)=0
$$

by a well-known result of Lebesgue. In the special case that $L$ has measure zero, we define $L_{\varepsilon}:=\emptyset$, hence $L_{\varepsilon}^{*}:=\emptyset$.

Observe that

$$
\mathcal{G}_{g} \cap \varphi(F) \backslash \varphi\left(L_{\varepsilon}^{*}\right) \subset \varphi\left(\varphi^{-1}\left(\mathcal{G}_{g}\right) \cap F \backslash L_{\varepsilon}^{*}\right)=\varphi\left(L \backslash L_{\varepsilon}^{*}\right)
$$

hence

$$
\begin{aligned}
\mathcal{H}^{n}\left(\mathcal{G}_{g} \cap \varphi(F) \backslash \varphi\left(L_{\varepsilon}^{*}\right)\right) & \leq \mathcal{H}^{n}\left(\varphi\left(L \backslash L_{\varepsilon}^{*}\right)\right) \\
& \leq \int_{L \backslash L_{\varepsilon}^{*}} J_{n} \varphi d \mathcal{L}^{n} \\
& \leq(\operatorname{Lip} \varphi)^{n} \mathcal{L}\left(L \backslash L_{\varepsilon}^{*}\right) \\
& \leq \varepsilon(\operatorname{Lip} \varphi)^{n}
\end{aligned}
$$


by the area formula (compare [F, §3.2.], [S, §8]), (3.1), (3.2) and (3.3). It follows that

$$
\mathcal{H}^{n}\left(\mathcal{G}_{g} \cap \varphi(F) \backslash \bigcup_{j=1}^{\infty} \varphi\left(L_{1 / j}^{*}\right)\right)=0 .
$$

Thus, to prove Theorem 2.5, it suffices to show that

$$
\varphi\left(L_{\varepsilon}^{*}\right) \text { is an }\left(\mathcal{H}^{n}, n\right) \text { rectifiable set of class } C^{3}
$$

for all $\varepsilon>0$.

3.2. Further notation. Let us consider the projection

$$
\Pi: \mathbb{R}^{N} \rightarrow \mathbb{R}^{N-n}, \quad\left(x_{1}, \ldots, x_{N}\right) \mapsto\left(x_{n+1}, \ldots, x_{N}\right) .
$$

Moreover set

$$
\begin{gathered}
\mathcal{R}_{s}^{(0)}(\sigma):=g(\lambda(\sigma))-g(\lambda(s))-\sum_{i=1}^{n} D_{i} g(\lambda(s))\left[\varphi^{i}(\sigma)-\varphi^{i}(s)\right]+ \\
-\frac{1}{2} \sum_{i, j=1}^{n} D_{i j}^{2} g(\lambda(s))\left[\varphi^{i}(\sigma)-\varphi^{i}(s)\right]\left[\varphi^{j}(\sigma)-\varphi^{j}(s)\right], \\
\mathcal{R}_{i ; s}^{(1)}(\sigma):=D_{i} g(\lambda(\sigma))-D_{i} g(\lambda(s))-\sum_{j=1}^{n} D_{i j}^{2} g(\lambda(s))\left[\varphi^{j}(\sigma)-\varphi^{j}(s)\right], \\
\mathcal{R}_{i j ; s}^{(2)}(\sigma):=D_{i j}^{2} g(\lambda(\sigma))-D_{i j}^{2} g(\lambda(s)) .
\end{gathered}
$$

For $h=1,2$, let $G_{h}$ denote the $n \times n$ matrix field such that

$$
\left[G_{h}(t)\right]_{i}^{j}:=G_{h, i}^{j}(t), \quad t \in \mathbb{R}^{n} \quad(i, j=1, \ldots, n) .
$$

Also let $H$ be the $n^{2} \times n$ matrix field defined by

$$
[H(t)]_{i j}^{k}:=H_{i j}^{k}(t), \quad t \in \mathbb{R}^{n} \quad(i, j, k=1, \ldots, n)
$$

where the couples $i j$ (indexing the rows) are ordered lexicographically.

Then consider the $\left(n+n^{2}\right) \times\left(n+n^{2}\right)$ matrix field

$$
M:=\left[\begin{array}{cc}
G_{1} & 0 \\
H & G_{1} \otimes G_{2}
\end{array}\right]
$$

where the symbol $\otimes$ denotes the Kronecker product of matrices, [HJ, Sect. $4.2]$.

For $l=1, \ldots, N-n$, let $D^{2} g^{l}$ denote the $\mathbb{R}^{n^{2}}$-valued field such that

$$
\left[D^{2} g^{l}(t)\right]^{i j}:=D_{i j}^{2} g^{l}(t), \quad t \in \mathbb{R}^{n} \quad(i, j=1, \ldots, n)
$$

where the lexicographical order is assumed.

Finally, given a matrix $X$ and a index $k$, denote by

$$
R_{k}(X), C_{k}(X)
$$

the $k$-th row of $X$ and $k$-th column of $X$, respectively. 
4. The Derivatives of $g$ In terms of $\left\{G_{1}, G_{2}, H\right\}$

(Under THE ASSUMPtions of TheOREM 2.5 , With $\gamma=\{1, \ldots, n\}$ )

Proposition 4.1. Let $l \in\{1, \ldots, N-n\}$ and $s \in L_{\varepsilon}^{*}$. Then

$$
M(s)\left(D g^{l}(\lambda(s)), D^{2} g^{l}(\lambda(s))\right)^{T}=\left(G_{1}^{n+l}(s), H^{n+l}(s)\right)^{T}
$$

where $G_{1}^{n+l}$ and $H^{n+l}$ are the the vector fields defined as follows:

$$
G_{1}^{n+l}:=\left(G_{1,1}^{n+l}, \ldots, G_{1, n}^{n+l}\right)
$$

and

$$
H^{n+l}:=\left[H_{i j}^{n+l}\right]_{i, j=1}^{n} \quad \text { (in lexicographical order). }
$$

Proof. First of all, observe that

$$
g(\lambda(t))=\Pi \varphi(t)
$$

for all $t \in \varphi^{-1}\left(\mathcal{G}_{g}\right)$. Since $L_{\varepsilon}^{*} \subset A$ the two members of this equality are both differentiable at $s$. Moreover $s$ is a limit point of $L_{\varepsilon} \subset \varphi^{-1}\left(\mathcal{G}_{g}\right)$. It follows that

$$
\sum_{j=1}^{n} D_{j} g(\lambda(s)) D_{i} \varphi^{j}(s)=\Pi D_{i} \varphi(s) \quad(i=1, \ldots, n)
$$

namely

$$
\sum_{j=1}^{n} D_{j} g(\lambda(s)) c_{1, i}(s) G_{1, i}^{j}(s)=c_{1, i}(s) \Pi G_{1, i}(s) \quad(i=1, \ldots, n)
$$

by $(1.1)$. Since $c_{1, i}(s) \neq 0(i=1, \ldots, n)$, we get

$$
\sum_{j=1}^{n} D_{j} g^{l}(\lambda(s)) G_{1, i}^{j}(s)=G_{1, i}^{n+l}(s) \quad(i=1, \ldots, n)
$$

i.e.

$$
G_{1}(s) D g^{l}(\lambda(s))=G_{1}^{n+l}(s) .
$$

By the same argument as above, we can differentiate (4.2) and obtain

$$
\sum_{j, k=1}^{n} D_{j k}^{2} g^{l}(\lambda(s)) D_{m} \varphi^{k}(s) G_{1, i}^{j}(s)+\sum_{j=1}^{n} D_{j} g^{l}(\lambda(s)) D_{m} G_{1, i}^{j}(s)=D_{m} G_{1, i}^{n+l}(s)
$$

for all $i, m=1, \ldots, n$. By $(1.2)$

$$
\begin{aligned}
& \sum_{j, k=1}^{n} D_{j k}^{2} g^{l}(\lambda(s)) c_{2, m}(s) G_{2, m}^{k}(s) G_{1, i}^{j}(s)+ \\
& \quad+\sum_{j=1}^{n} D_{j} g^{l}(\lambda(s)) c_{2, m}(s) H_{i m}^{j}(s)=c_{2, m}(s) H_{i m}^{n+l}(s)
\end{aligned}
$$

for all $i, m=1, \ldots, n$, namely

$$
\left[G_{1}(s) \otimes G_{2}(s)\right] D^{2} g^{l}(\lambda(s))+H(s) D g^{l}(\lambda(s))=H^{n+l}(s) .
$$


We conclude by observing that the system of equalities (4.3) and (4.4) is equivalent to (4.1).

In this result we investigate the properties of the matrix field $t \mapsto M(t)^{-1}$.

Proposition 4.2. Let $s \in A$ be such that $D \lambda(s)$ is nonsingular (e.g. $s \in F$ ). Then there exists a nontrivial ball $B$, centered at $s$, such that

- For all $t \in B$, the matrices $G_{1}(t), G_{2}(t)$ and $M(t)$ are invertible and

$$
M(t)^{-1}=\left[\begin{array}{cc}
G_{1}(t)^{-1} & 0 \\
-\left[G_{1}(t)^{-1} \otimes G_{2}(t)^{-1}\right] H(t) G_{1}(t)^{-1} & G_{1}(t)^{-1} \otimes G_{2}(t)^{-1}
\end{array}\right]
$$

- The map

$$
t \mapsto M(t)^{-1}, \quad t \in B
$$

is Lipschitz.

Proof. One has

$$
D \lambda(s)=\left[\prod_{i=1}^{n} c_{1, i}(s)\right] G_{1}(s)^{T}=\left[\prod_{i=1}^{n} c_{2, i}(s)\right] G_{2}(s)^{T}
$$

by (1.1), hence $G_{1}(s)$ and $G_{2}(s)$ are nonsingular. Moreover one has

$$
\operatorname{det} M=\operatorname{det} G_{1} \operatorname{det}\left(G_{1} \otimes G_{2}\right)=\left(\operatorname{det} G_{1}\right)^{n+1}\left(\operatorname{det} G_{2}\right)^{n}
$$

by [HJ, Sect. 4.2, Problem 1]. Thus

$$
\operatorname{det} M(s) \neq 0 \text {. }
$$

Since the function $t \mapsto \operatorname{det} M(t)$ is continuous, there exists a nontrivial ball $B$ centered at $s$ and such that

$$
|\operatorname{det} M(t)| \geq \frac{|\operatorname{det} M(s)|}{2}>0
$$

for all $t \in B$. As a consequence, $M(t)$ is invertible at every $t \in B$. The formula (4.5) follows at once observing that, for $t \in B$, the matrix $M(t)^{-1}$ has to be of the form (recall (4.6))

$$
\left[\begin{array}{cc}
G_{1}(t)^{-1} & 0 \\
X(t) & {\left[G_{1}(t) \otimes G_{2}(t)\right]^{-1}}
\end{array}\right]
$$

with $X(t)$ satisfying

$$
H(t) G_{1}(t)^{-1}+\left[G_{1}(t) \otimes G_{2}(t)\right] X(t)=0
$$

and finally recalling that

$$
\left[G_{1}(t) \otimes G_{2}(t)\right]^{-1}=G_{1}(t)^{-1} \otimes G_{2}(t)^{-1}
$$

compare [HJ, Corollary 4.2.11]. This concludes the proof of the first claim. The second one follows by observing that the entries of $M$ are Lipschitz. 


\section{Whitney-TyPe EStimates}

(Under The ASSUMPtions of TheOREM 2.5 , With $\gamma=\{1, \ldots, n\}$ )

Proposition 5.1. Let $s \in L_{\varepsilon}^{*}$ and $t \in A \cap \varphi^{-1}\left(\mathcal{G}_{g}\right)$ be such that

$$
\mathcal{H}^{1}([s ; t] \backslash A)=0
$$

where $[s ; t]$ denotes the segment joining $s$ and $t$. Then the following estimate holds

$$
\left\|\mathcal{R}_{s}^{(0)}(t)\right\| \leq\left(\sup _{[s ; t]}\left\|c_{1}\right\|\right)\left(\sup _{[s ; t]}\left\|c_{2}\right\|\right) \Lambda_{s}\|t-s\|^{3}
$$

where

$$
c_{1}:=\left(c_{1,1}, \ldots, c_{1, n}\right), \quad c_{2}:=\left(c_{2,1}, \ldots, c_{2, n}\right)
$$

and $\Lambda_{s}$ is a constant not depending on $t$.

Proof. First of all, observe that:

- Since $s, t \in \varphi^{-1}\left(\mathcal{G}_{g}\right)$ one has $g(\lambda(s))=\Pi \varphi(s)$ and $g(\lambda(t))=\Pi \varphi(t)$;

- Consider the following parametrization of $[s ; t]$

$$
\sigma:[0,1] \rightarrow \mathbb{R}^{n}, \quad \rho \mapsto s+\rho(t-s) .
$$

Then the function $\rho \mapsto \varphi(\sigma(\rho))$ is Lipschitz, hence it is differentiable almost everywhere in $[0,1]$. Moreover the assumption (5.1) implies that

$$
(\varphi \circ \sigma)^{\prime}(\rho)=\sum_{i_{1}=1}^{n}\left(t^{i_{1}}-s^{i_{1}}\right) D_{i_{1}} \varphi(\sigma(\rho))
$$

at a.e. $\rho \in[0,1]$.

Recalling also (1.1), we obtain

$$
\begin{aligned}
\mathcal{R}_{s}^{(0)}(t)= & \Pi \varphi(t)-\Pi \varphi(s)-\sum_{i=1}^{n} D_{i} g(\lambda(s))\left[\varphi^{i}(t)-\varphi^{i}(s)\right]+ \\
& -\frac{1}{2} \sum_{i, j=1}^{n} D_{i j}^{2} g(\lambda(s))\left[\varphi^{i}(t)-\varphi^{i}(s)\right]\left[\varphi^{j}(t)-\varphi^{j}(s)\right] \\
= & \sum_{h=1}^{n}\left(t^{h}-s^{h}\right) \int_{0}^{1}\left\{\Pi D_{h} \varphi(\sigma(\rho))-\sum_{i=1}^{n} D_{i} g(\lambda(s)) D_{h} \varphi^{i}(\sigma(\rho))+\right. \\
& \left.-\sum_{i, j=1}^{n} D_{i j}^{2} g(\lambda(s))\left[\varphi^{i}(\sigma(\rho))-\varphi^{i}(s)\right] D_{h} \varphi^{j}(\sigma(\rho))\right\} d \rho
\end{aligned}
$$

that is

$$
\mathcal{R}_{s}^{(0)}(t)=\sum_{h=1}^{n}\left(t^{h}-s^{h}\right) \int_{0}^{1} c_{1, h}(\sigma(\rho)) \Phi_{s, h}(\sigma(\rho)) d \rho
$$


where $\Phi_{s, h}$ denotes the Lipschitz map defined as follows

$$
\Phi_{s, h}:=\Pi G_{1, h}-\sum_{i=1}^{n} D_{i} g(\lambda(s)) G_{1, h}^{i}-\sum_{i, j=1}^{n} D_{i j}^{2} g(\lambda(s))\left[\varphi^{i}-\varphi^{i}(s)\right] G_{1, h}^{j}
$$

Now, since $\Phi_{s, h} \circ \sigma$ is Lipschitz, it is differentiable almost everywhere in $[0,1]$ and

$$
\left(\Phi_{s, h} \circ \sigma\right)^{\prime}=\sum_{k=1}^{n}\left(t^{k}-s^{k}\right)\left(D_{k} \Phi_{s, h}\right) \circ \sigma .
$$

Moreover $\Phi_{s, h}(s)=0$, by (4.2). By (5.3) and recalling (1.2), we get

$$
\begin{aligned}
\Phi_{s, h}(\sigma(\rho)) & =\Phi_{s, h}(\sigma(\rho))-\Phi_{s, h}(s)=\int_{0}^{\rho}\left(\Phi_{s, h} \circ \sigma\right)^{\prime} \\
& =\sum_{k=1}^{n}\left(t^{k}-s^{k}\right) \int_{0}^{\rho}\left(D_{k} \Phi_{s, h}\right) \circ \sigma \\
& =\sum_{k=1}^{n}\left(t^{k}-s^{k}\right) \int_{0}^{\rho}\left(c_{2, k} \circ \sigma\right)\left(\Psi_{s, h k} \circ \sigma\right)
\end{aligned}
$$

where $\Psi_{s, h k}$ is the Lipschitz map defined by

$$
\begin{aligned}
\Psi_{s, h k}:=\Pi H_{h k} & -\sum_{i=1}^{n} D_{i} g(\lambda(s)) H_{h k}^{i}+ \\
& -\sum_{i, j=1}^{n} D_{i j}^{2} g(\lambda(s))\left\{G_{2, k}^{i} G_{1, h}^{j}+\left[\varphi^{i}-\varphi^{i}(s)\right] H_{h k}^{j}\right\} .
\end{aligned}
$$

Observe that

$$
\begin{aligned}
\Psi_{s, h k}(s) & =\Pi H_{h k}(s)-\sum_{i=1}^{n} D_{i} g(\lambda(s)) H_{h k}^{i}(s)-\sum_{i, j=1}^{n} D_{i j}^{2} g(\lambda(s)) G_{2, k}^{i}(s) G_{1, h}^{j}(s) \\
& =0
\end{aligned}
$$

by (4.4). Hence (for all $r \in[0,1]$ )

$$
\begin{aligned}
\left\|\Psi_{s, h k}(\sigma(r))\right\| & =\left\|\Psi_{s, h k}(\sigma(r))-\Psi_{s, h k}(s)\right\| \leq\|\sigma(r)-s\| \operatorname{Lip} \Psi_{s, h k} \\
& =r\|t-s\| \operatorname{Lip} \Psi_{s, h k} \\
& \leq\|t-s\| \Lambda_{s}
\end{aligned}
$$

with

$$
\Lambda_{s}:=\max _{h, k=1, \ldots, n}\left(\operatorname{Lip} \Psi_{s, h k}\right) .
$$

Recalling (5.4), we obtain

$$
\left\|\Phi_{s, h}(\sigma(\rho))\right\| \leq\left(\sup _{[s ; t]}\left\|c_{2}\right\|\right) \Lambda_{s}\|t-s\|^{2} .
$$

The conclusion follows at once from (5.2). 
Proposition 5.2. Let $s \in L_{\varepsilon}^{*}$. Then there exists a nontrivial ball B, centered at $s$, such that

$$
\left\|\mathcal{R}_{i ; s}^{(1)}(t)\right\| \leq\left(\sup _{[s ; t]}\left\|c_{2}\right\|\right) \Sigma_{s}\|t-s\|^{2} \quad(i=1, \ldots, n)
$$

for all $t \in L_{\varepsilon}^{*} \cap B$ such that (5.1) is satisfied, where $c_{2}$ is defined as in Proposition 5.1 while $\Sigma_{s}$ is a constant not depending on $t$ and $i$.

Proof. Since $s \in L_{\varepsilon}^{*} \subset F$, there exists a ball $B$ as in Proposition 4.2. Consider

$$
t \in L_{\varepsilon}^{*} \cap B
$$

such that (5.1) is satisfied. Then (for $l=1, \ldots, N-n$ )

$$
\begin{aligned}
{\left[\mathcal{R}_{i ; s}^{(1)}(t)\right]^{l}=} & D_{i} g^{l}(\lambda(t))-D_{i} g^{l}(\lambda(s))-\sum_{j=1}^{n} D_{i j}^{2} g^{l}(\lambda(s))\left[\varphi^{j}(t)-\varphi^{j}(s)\right] \\
= & R_{i}\left(G_{1}(t)^{-1}\right) \bullet G_{1}^{n+l}(t)-R_{i}\left(G_{1}(s)^{-1}\right) \bullet G_{1}^{n+l}(s)+ \\
& -\sum_{j=1}^{n} D_{i j}^{2} g^{l}(\lambda(s))\left[\varphi^{j}(t)-\varphi^{j}(s)\right]
\end{aligned}
$$

by Proposition 4.1 and Proposition 4.2. Moreover, if $\sigma$ is the parametrization of $[s ; t]$ defined above, the function

$$
\Pi: \rho \mapsto R_{i}\left(G_{1}(\sigma(\rho))^{-1}\right) \bullet G_{1}^{n+l}(\sigma(\rho)), \quad \rho \in[0,1]
$$

is Lipschitz, hence it is differentiable almost everywhere in $[0,1]$. Recalling (5.1) and denoting with $G_{1}^{-1}$ the map $r \mapsto G_{1}(r)^{-1}$ (by a convenient abuse of notation), we obtain

$$
\Pi^{\prime}(\rho)=\sum_{q=1}^{n}\left(t^{q}-s^{q}\right)\left\{R_{i}\left(D_{q} G_{1}^{-1}\right) \bullet G_{1}^{n+l}+R_{i}\left(G_{1}^{-1}\right) \bullet D_{q} G_{1}^{n+l}\right\}(\sigma(\rho))
$$

for a.e. $\rho \in[0,1]$. By the well-known formula for the derivative of the inverse matrix field, compare [HJ, (6.5.7)], it follows that

$$
\begin{aligned}
\Pi^{\prime}(\rho)= & \sum_{q=1}^{n}\left(t^{q}-s^{q}\right)\left\{R_{i}\left(G_{1}^{-1}\right) \bullet D_{q} G_{1}^{n+l}+\right. \\
& \left.-R_{i}\left[G_{1}^{-1}\left(D_{q} G_{1}\right) G_{1}^{-1}\right] \bullet G_{1}^{n+l}\right\}(\sigma(\rho)) \\
= & \sum_{m, q=1}^{n}\left(t^{q}-s^{q}\right)\left\{\left[G_{1}^{-1}\right]_{i}^{m} D_{q} G_{1, m}^{n+l}-\left[G_{1}^{-1}\left(D_{q} G_{1}\right) G_{1}^{-1}\right]_{i}^{m} G_{1, m}^{n+l}\right\}(\sigma(\rho)) \\
= & \sum_{m, q=1}^{n}\left(t^{q}-s^{q}\right)\left\{\left[G_{1}^{-1}\right]_{i}^{m} D_{q} G_{1, m}^{n+l}\right. \\
& \left.-\sum_{h, k=1}^{n}\left[G_{1}^{-1}\right]_{i}^{h}\left(D_{q} G_{1, h}^{k}\right)\left[G_{1}^{-1}\right]_{k}^{m} G_{1, m}^{n+l}\right\}(\sigma(\rho))
\end{aligned}
$$


for a.e. $\rho \in[0,1]$. Recalling $(1.2)$, we get

$$
\begin{aligned}
\Pi^{\prime}(\rho)= & \sum_{m, q=1}^{n} c_{2, q}(\sigma(\rho))\left(t^{q}-s^{q}\right)\left\{\left[G_{1}^{-1}\right]_{i}^{m} H_{m q}^{n+l}+\right. \\
& \left.-\sum_{h, k=1}^{n}\left[G_{1}^{-1}\right]_{i}^{h} H_{h q}^{k}\left[G_{1}^{-1}\right]_{k}^{m} G_{1, m}^{n+l}\right\}(\sigma(\rho))
\end{aligned}
$$

for a.e. $\rho \in[0,1]$. It follows that

$$
\left[\mathcal{R}_{i ; s}^{(1)}(t)\right]^{l}=\sum_{q=1}^{n}\left(t^{q}-s^{q}\right) \int_{0}^{1} c_{2, q}(\sigma(\rho)) \Theta_{q ; s}^{l}(\sigma(\rho)) d \rho
$$

where $\Theta_{q ; s}^{l}: B \rightarrow \mathbb{R}$ is the function defined as

$\Theta_{q ; s}^{l}:=\sum_{m=1}^{n}\left\{\left[G_{1}^{-1}\right]_{i}^{m} H_{m q}^{n+l}-\sum_{h, k=1}^{n}\left[G_{1}^{-1}\right]_{i}^{h} H_{h q}^{k}\left[G_{1}^{-1}\right]_{k}^{m} G_{1, m}^{n+l}-D_{i m}^{2} g^{l}(\lambda(s)) G_{2, q}^{m}\right\}$.

One has

$$
\begin{aligned}
D_{i m}^{2} g^{l}(\lambda(s))= & \sum_{c, d=1}^{n}\left[G_{1}(s)^{-1} \otimes G_{2}(s)^{-1}\right]_{i m}^{c d} H_{c d}^{n+l}(s)+ \\
& -\sum_{b, c, d, e=1}^{n}\left[G_{1}(s)^{-1} \otimes G_{2}(s)^{-1}\right]_{i m}^{c d} H_{c d}^{b}(s)\left[G_{1}(s)^{-1}\right]_{b}^{e} G_{1, e}^{n+l}(s) \\
= & \sum_{c, d=1}^{n}\left[G_{1}(s)^{-1}\right]_{i}^{c}\left[G_{2}(s)^{-1}\right]_{m}^{d} H_{c d}^{n+l}(s)+ \\
& -\sum_{b, c, d, e=1}^{n}\left[G_{1}(s)^{-1}\right]_{i}^{c}\left[G_{2}(s)^{-1}\right]_{m}^{d} H_{c d}^{b}(s)\left[G_{1}(s)^{-1}\right]_{b}^{e} G_{1, e}^{n+l}(s)
\end{aligned}
$$

by Proposition 4.1 and Proposition 4.2. Hence the following equality holds

$$
\begin{aligned}
\sum_{m=1}^{n} D_{i m}^{2} g^{l}(\lambda(s)) G_{2, q}^{m}(s)= & \sum_{c, d=1}^{n}\left[G_{1}(s)^{-1}\right]_{i}^{c} H_{c d}^{n+l}(s) \delta_{d q}+ \\
& -\sum_{b, c, d, e=1}^{n}\left[G_{1}(s)^{-1}\right]_{i}^{c} H_{c d}^{b}(s)\left[G_{1}(s)^{-1}\right]_{b}^{e} G_{1, e}^{n+l}(s) \delta_{d q} \\
= & \sum_{c=1}^{n}\left[G_{1}(s)^{-1}\right]_{i}^{c} H_{c q}^{n+l}(s)+ \\
& -\sum_{b, c, e=1}^{n}\left[G_{1}(s)^{-1}\right]_{i}^{c} H_{c q}^{b}(s)\left[G_{1}(s)^{-1}\right]_{b}^{e} G_{1, e}^{n+l}(s)
\end{aligned}
$$

namely

$$
\Theta_{q ; s}^{l}(s)=0
$$


Moreover $\Theta_{q ; s}^{l}$ is Lipschitz, by Proposition 4.2. Then, if define

$$
\Sigma_{s}:=(N-n) \max _{\substack{q=1, \ldots, n \\ l=1, \ldots, N-n}}\left(\operatorname{Lip} \Theta_{q ; s}^{l}\right)
$$

we get

$$
\left|\Theta_{q ; s}^{l}(\sigma(\rho))\right|=\left|\Theta_{q ; s}^{l}(\sigma(\rho))-\Theta_{q ; s}^{l}(s)\right| \leq \frac{\Sigma_{s}}{N-n} \rho\|t-s\| \leq \frac{\Sigma_{s}}{N-n}\|t-s\|
$$

for all $q=1, \ldots, n$, for all $l=1, \ldots, N-n$ and for all $\rho \in[0,1]$. From (5.5) it finally follows that

$$
\left\|\mathcal{R}_{i ; s}^{(1)}(t)\right\| \leq \sum_{l=1}^{N-n}\left|\left[\mathcal{R}_{i ; s}^{(1)}(t)\right]^{l}\right| \leq\left(\sup _{[s ; t]}\left\|c_{2}\right\|\right) \Sigma_{s}\|t-s\|^{2} .
$$

The estimate of the second order remainder term is established in the following result, which is an immediate conseguence of Proposition 4.2 and (4.1).

Proposition 5.3. Let $s \in L_{\varepsilon}^{*}$. Then there exists a nontrivial ball B, centered at $s$, such that

$$
\left\|\mathcal{R}_{i j ; s}^{(2)}(t)\right\|=\left\|D_{i j}^{2} g(\lambda(t))-D_{i j}^{2} g(\lambda(s))\right\| \leq \Gamma_{s}\|t-s\| \quad(i, j=1, \ldots, n)
$$

for all $t \in L_{\varepsilon}^{*} \cap B$, where $\Gamma_{s}$ is a constant not depending on $t$ and $i, j$.

\section{Proof of Theorem 2.5}

As we pointed out in Section 3.1, we are reduced to prove that $\varphi\left(L_{\varepsilon}^{*}\right)$ is an $\left(\mathcal{H}^{n}, n\right)$ rectifiable set of class $C^{3}$ (for all $\varepsilon>0$ ).

For each positive integer $h$, define $\Gamma_{\varepsilon, h}$ as the set of $s \in L_{\varepsilon}^{*}$ such that

$$
\left\|\mathcal{R}_{s}^{(0)}(t)\right\| \leq h\|\lambda(t)-\lambda(s)\|^{3}
$$

and

$$
\left\|\mathcal{R}_{i ; s}^{(1)}(t)\right\| \leq h\|\lambda(t)-\lambda(s)\|^{2}, \quad\left\|\mathcal{R}_{i j ; s}^{(2)}(t)\right\| \leq h\|\lambda(t)-\lambda(s)\|
$$

for all $i, j=1, \ldots, n$ and for all $t \in L_{\varepsilon}^{*}$ satisfying

$$
\|t-s\| \leq \frac{1}{h}
$$

Proposition 6.1. One has

$$
\bigcup_{h} \Gamma_{\varepsilon, h}=L_{\varepsilon}^{*}
$$


Proof. The inclusion

$$
\bigcup_{h} \Gamma_{\varepsilon, h} \subset L_{\varepsilon}^{*}
$$

is obvious. In order to prove the opposite inclusion, consider $s \in L_{\varepsilon}^{*}$ and let $U$ and $V$ be as in Remark 2.4. Observe that

$$
\begin{aligned}
\|t-s\| & =\left\|(\lambda \mid U)^{-1}(\lambda(t))-(\lambda \mid U)^{-1}(\lambda(s))\right\| \\
& \leq \operatorname{Lip}(\lambda \mid U)^{-1}\|\lambda(t)-\lambda(s)\|
\end{aligned}
$$

for all $t \in U$.

Since $s \in A^{\prime}$, there exists a non-trivial ball $B$ centered at $s$ such that

$$
B \subset U, \quad \mathcal{L}^{n}(B \backslash A)=0 .
$$

By shrinking, if need be, we may also assume that $B$ is as in the claims of Proposition 5.2 and Proposition 5.3.

We now recall the following fact, proved in [D5]: given a null-measure subset $Z$ of $\mathbb{R}^{n}$ and $s \in \mathbb{R}^{n}$, one has

$$
\mathcal{H}^{1}(Z \cap[s ; t])=0
$$

for a.e. $t \in \mathbb{R}^{n}$.

For $Z:=B \backslash A$, we get

$$
\mathcal{H}^{1}([s ; t] \backslash A)=\mathcal{H}^{1}(Z \cap[s ; t])=0
$$

for a.e. $t \in B$. Then Proposition 5.1 yields

$$
\left\|\mathcal{R}_{s}^{(0)}(t)\right\| \leq C\|t-s\|^{3}
$$

for a.e. $t \in B \cap \varphi^{-1}\left(\mathcal{G}_{g}\right)$, where $C$ is a suitable number which does not depend on $t$. By continuity we get

$$
\left\|\mathcal{R}_{s}^{(0)}(t)\right\| \leq C\|t-s\|^{3}
$$

for all $t \in B \cap \varphi^{-1}\left(\mathcal{G}_{g}\right)$. Recalling (6.3) we conclude that

$$
\left\|\mathcal{R}_{s}^{(0)}(t)\right\| \leq C_{0}\|\lambda(t)-\lambda(s)\|^{3}, \quad C_{0}:=C\left[\operatorname{Lip}(\lambda \mid U)^{-1}\right]^{3}
$$

for all $t \in B \cap \varphi^{-1}\left(\mathcal{G}_{g}\right)$. Analogously, we can use Proposition 5.2, Proposition 5.3 and (6.3) to deduce the existence of two numbers $C_{1}$ and $C_{2}$ which do not depend on $t$ and are such that

$$
\left\|\mathcal{R}_{i ; s}^{(1)}(t)\right\| \leq C_{1}\|\lambda(t)-\lambda(s)\|^{2} \quad(i=1, \ldots, n)
$$

and

$$
\left\|\mathcal{R}_{i j ; s}^{(2)}(t)\right\| \leq C_{2}\|\lambda(t)-\lambda(s)\| \quad(i, j=1, \ldots, n)
$$

for all $t \in L_{\varepsilon}^{*} \cap B$.

Hence

$$
s \in \Gamma_{\varepsilon, h}
$$


provided $h$ is big enough.

From Proposition 6.1 it follows that

$$
\varphi\left(L_{\varepsilon}^{*}\right)=\bigcup_{h} \varphi\left(\Gamma_{\varepsilon, h}\right)
$$

hence it will be enough to verify that

$$
\varphi\left(\Gamma_{\varepsilon, h}\right) \text { is an }\left(\mathcal{H}^{n}, n\right) \text { rectifiable set of class } C^{3}
$$

for all $\varepsilon$ and $h$.

To prove this claim, we first consider a countable measurable covering $\left\{Q_{l}\right\}_{l=1}^{\infty}$ of $\Gamma_{\varepsilon, h}$ such that

for all $l$, and define

$$
\operatorname{diam} Q_{l} \leq \frac{1}{h}
$$

$$
F_{l}:=\overline{\lambda\left(\Gamma_{\varepsilon, h} \cap Q_{l}\right)}
$$

If $\xi, \eta \in F_{l}$, then there exist two sequences

$$
\left\{s_{k}\right\},\left\{t_{k}\right\} \subset \Gamma_{\varepsilon, h} \cap Q_{l}
$$

such that

$$
\lim _{k} \lambda\left(s_{k}\right)=\xi, \quad \lim _{k} \lambda\left(t_{k}\right)=\eta .
$$

By (6.1) and (6.2), for all $k$, one has

$$
\left\|\mathcal{R}_{s_{k}}^{(0)}\left(t_{k}\right)\right\| \leq h\left\|\lambda\left(t_{k}\right)-\lambda\left(s_{k}\right)\right\|^{3}
$$

and

$$
\left\|\mathcal{R}_{i, s_{k}}^{(1)}\left(t_{k}\right)\right\| \leq h\left\|\lambda\left(t_{k}\right)-\lambda\left(s_{k}\right)\right\|^{2}, \quad\left\|\mathcal{R}_{i j, s_{k}}^{(2)}\left(t_{k}\right)\right\| \leq h\left\|\lambda\left(t_{k}\right)-\lambda\left(s_{k}\right)\right\|
$$

for all $i, j=1, \ldots, n$. Letting $k \rightarrow \infty$, we obtain

$$
\begin{gathered}
\left\|g(\eta)-g(\xi)-\sum_{i=1}^{n} D_{i} g(\xi)\left(\eta^{i}-\xi^{i}\right)-\frac{1}{2} \sum_{i, j=1}^{n} D_{i j}^{2} g(\xi)\left(\eta^{i}-\xi^{i}\right)\left(\eta^{j}-\xi^{j}\right)\right\| \leq h\|\eta-\xi\|^{3}, \\
\left\|D_{i} g(\eta)-D_{i} g(\xi)-\sum_{j=1}^{n} D_{i j}^{2} g(\xi)\left(\eta^{j}-\xi^{j}\right)\right\| \leq h\|\eta-\xi\|^{2} \quad(i=1, \ldots, n)
\end{gathered}
$$

and

$$
\left\|D_{i j}^{2} g(\eta)-D_{i j}^{2} g(\xi)\right\| \leq h\|\eta-\xi\| \quad(i, j=1, \ldots, n)
$$

for all $\xi, \eta \in F_{l}$. By the Whitney extension Theorem [St, Ch. VI, §2.3] it follows that each $g \mid F_{l}$ can be extended to a map in $C^{2,1}\left(\mathbb{R}^{n}, \mathbb{R}^{N-n}\right)$. Then the Lusin type result $[\mathrm{F}, \S 3.1 .15]$ implies that $\varphi\left(\Gamma_{\varepsilon, h} \cap Q_{l}\right)$ is an $\left(\mathcal{H}^{n}, n\right)$ rectifiable set of class $C^{3}$. Finally, claim (6.4) follows observing that

$$
\varphi\left(\Gamma_{\varepsilon, h}\right)=\bigcup_{l} \varphi\left(\Gamma_{\varepsilon, h} \cap Q_{l}\right) .
$$




\section{REFERENCES}

[A] G. Alberti, On the structure of singular sets of convex functions. Calc. Var. 2, 17-27 (1994).

[AO] G. Anzellotti, E. Ossanna, Singular sets of surfaces with generalized curvatures. Manuscripta Math. 86 (1995), 417-433.

[AS] G. Anzellotti, R. Serapioni, $\mathcal{C}^{k}$-rectifiable sets. J. reine angew. Math. 453 (1994), 1-20.

[AST] G. Anzellotti, R. Serapioni and I. Tamanini, Curvatures, Functionals, Currents. Indiana Univ. Math. J. 39 (1990), 617-669.

[BHS] B. Bojarski, P. Hajlasz, P. Strzelecki, Sard's theorem for mappings in Hölder and Sobolev spaces. Manuscripta Math. 118 (2005), n. 3, 383-397.

[CLSW] F.H. Clarke, Yu.S. Ledyaev, R.J. Stern, P.R. Wolenski, Nonsmooth analysis and control theory. Graduate Texts in Mathematics, Springer Verlag, 1998.

[D1] S. Delladio, Do Generalized Gauss Graphs Induce Curvature Varifolds? Boll. Un. Matem. Italiana 10-B (1996), 991-1017.

[D2] S. Delladio, A result about $C^{2}$-rectifiability of one-dimensional rectifiable sets. Application to a class of one-dimensional integral currents. Boll. Un. Matem. Italiana 10-B (2007), 237-252.

[D3] S. Delladio, A sufficient condition for the $C^{H}$-rectifiability of Lipschitz curves. J. Geom. Anal. 18 (2008), n. 3, 746-764.

[D4] S. Delladio, A class of one-dimensional geometric variational problems with energy involving the curvature and its derivatives. A geometric measure-theoretic approach. Ann. Mat. Pura ed Appl. 187 (2008), n. 4, 611-645.

[D5] S. Delladio, A sufficient condition for the $C^{2}$-rectifiability of the set of regular values (in the sense of Clarke) of a Lipschitz map. Boll. Un. Matem. Italiana (9) I (2008), n. 3, 695-707.

[F] H. Federer, Geometric Measure Theory. Springer-Verlag, 1969.

[Fu1] J.H.G. Fu, Some Remarks On Legendrian Rectifiable Currents. Manuscripta Math. 97 (1998), n. 2, 175-187.

[Fu2] J.H.G. Fu, Erratum to "Some Remarks On Legendrian Rectifiable Currents". Manuscripta Math. 113 (2004), n. 3, 397-401.

[HJ] R.A. Horn, C.R. Johnson, Topics in Matrix Analysis. Cambridge University Press, 1999.

[M] P. Mattila, Geometry of sets and measures in Euclidean spaces. Cambridge University Press, 1995. 
[S] L. Simon, Lectures on Geometric Measure Theory. Proceedings of the Centre for Mathematical Analysis, Canberra, Australia, vol. 3, 1984.

[St] E.M. Stein, Singular Integrals and Differentiability Properties of Functions. Princeton University Press, Princeton, 1970.

Department of Mathematics, University of Trento, Trento, Italy

E-mail address: silvano.delladio@unitn.it 